\title{
Circular Traffic Sign Detection in Natural Scenes
}

\author{
Qi Zhang1, Ning $\mathrm{He}^{2 *}$, Yue Chi ${ }^{1}$ \\ ${ }^{1}$ Beijing Key Laboratory of Information Services Engineering, Beijing Union University, Beijing 100101, \\ China. \\ 2 College of Information Technology, Beijing Union University, Beijing 100101, China. \\ * Corresponding author. Tel: +86-138-1115-6835; email: xxthening@buu.edu.cn \\ Manuscript submitted May 29, 2016; accepted January 5, 2017. \\ doi: $10.17706 /$ jcp.13.1.69-76
}

\begin{abstract}
We propose an approach for detecting circular traffic signs from images degraded by motion blur recorded in natural scenes. The first step enhances the image using the Laplace algorithm. The second step converts the image color space and identifies circular traffic signs using an adaptive threshold segmentation algorithm. The final step detects the sign area by considering circular and area features. Our experiments demonstrated that the method is robust and adaptable.
\end{abstract}

Keywords: Traffic sign, image segmentation, HSV space, adaptive threshold.

\section{Introduction}

Cyber car and driver assistance systems are becoming increasingly important in modern intelligent transportation research. Vehicle ownership has been rapidly increasing, so the traffic burden on urban highway networks has become more serious. Intelligent transportation systems (ITS) have emerged. Among these new technologies, traffic sign recognition has been extensively studied [1], [2]. A traffic sign recognition system has an on-board camera that captures images, then uses image detection to precisely determine the traffic sign position and deliver information to drivers. Traffic sign detection is an important component of traffic sign recognition.

Traffic sign recognition was first proposed in 1984 [3]. Many companies and research institutes have carried out research and development work in this field. Developments in computer and information technologies have led to great progress in traffic sign identification technologies. Existing methods generally preprocess relevant videos or images, and then segment the figure according to colors or shapes to produce the final extraction [4]. For circular signs, the Hough or radial symmetry transforms are typically used to extract the position.

The nature of traffic signs naturally lead to methods based on color threshold segmentation such as setting an RGB threshold to segment images. This method is simple to implement, but there are no correlations among the RGB components so it can be easily affected by natural light variations and produce incomplete traffic signs. To improve the color segmentation under the RGB model, a method for image segmentation based on chromatic aberrations was proposed [5]. Some researchers have used unrelated HIS space three-component detection for traffic signs, which weaken the influence of lighting conditions [6], [7]. The geometry of a traffic sign is not affected by light, so the method in [8] used the Canny operator to extract the edges of images, and then used the Hough transform to extract the traffic signs [8]. However, the method was not computationally efficient. Loy and Barnes [9], [10] considered the radial symmetry of traffic signs and 
proposed a radial symmetry transform algorithm. Zhu et al. [11] established color-shape pair for traffic signs, combining specific color segmentation results and shape features because the shape is not influenced by light, shade, or adjacent signs. However the method was slow and susceptible to the tilt angle. In normal light and the absence of other distractions, these algorithms performed well.

Traditional algorithms cannot adapt to complex natural scenes and all kinds of traffic signs. The primary objectives of our work were to extract, detect, and segment circular traffic signs. Circular traffic signs are prohibited and speed limit signs, and are mainly red. We first use an improved threshold segmentation algorithm, and then use a morphological method to extract the circular traffic sign and detect its location.

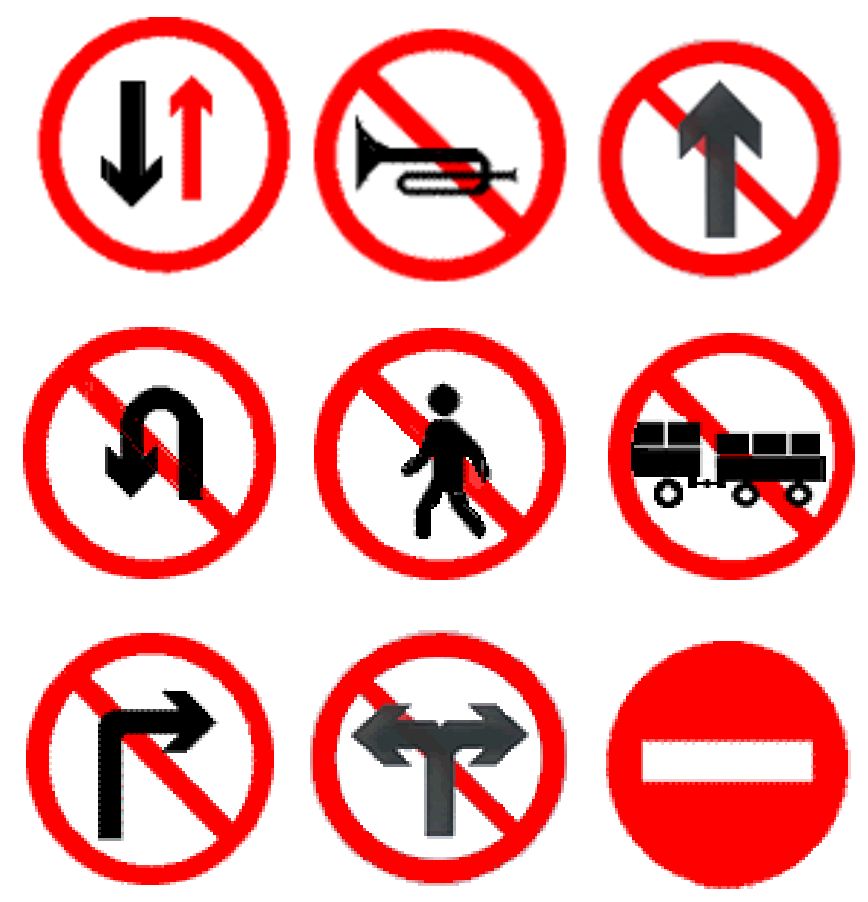

Fig. 1. Common circular traffic signs.

\section{Traffic Sign Segmentation}

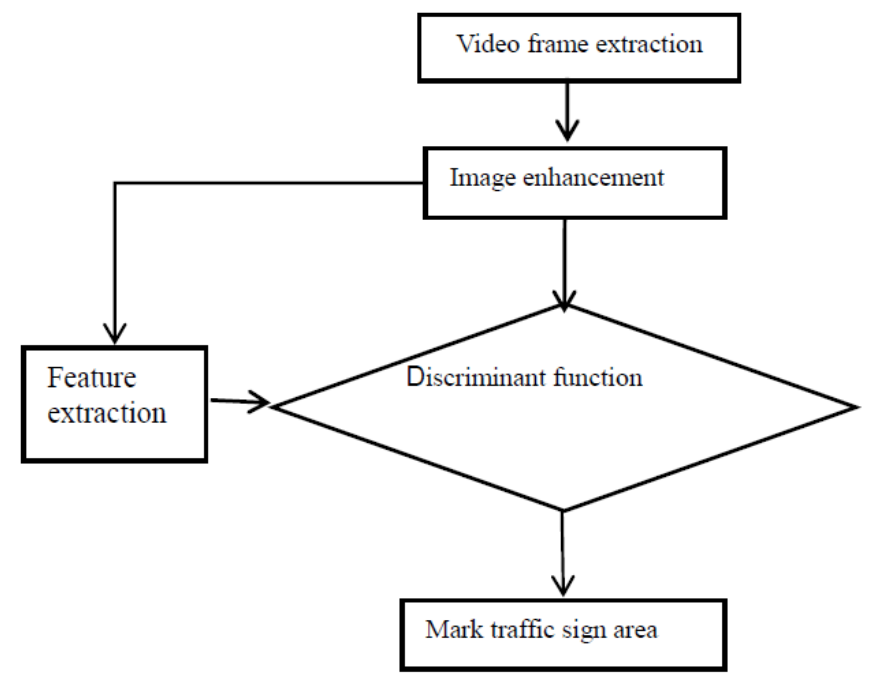

Fig. 2. Flowchart of the proposed traffic sign extraction method.

\subsection{Image Enhancement}

The video sequence to be processed is obtained when the vehicle is travelling, so there is often motion blur, 
which affects the image segmentation result [12]. Blurred images are essentially formed by a smoothing process applied to each pixel, its neighboring pixels, and the direction of the movement using a progressive average. This is called convolution. Thus, the first step is deconvolution. We use traditional motion blur methods to estimate the degradation function, and then apply the inverse function. However, this algorithm is time-consuming so the results are not accurate in real-time. If the blurring is not too substantial, the results obtained using the sharpness enhancement method can extract a more accurate segmentation. Conventional sharpening methods include the Roberts, Sobel, Prewitt, and Laplacian operators [13]. The image to be processed contains almost no isolated points or lines, but we must highlight object edges. The Laplacian operator can better highlight edge information, so we used it as a template operator to produce a clear image for segmentation.

\begin{tabular}{|l|l|l|}
\hline 0 & -1 & 0 \\
\hline-1 & 5 & -1 \\
\hline 0 & -1 & 0 \\
\hline
\end{tabular}

Fig. 3. Laplacian template.

The effect of this operator is shown in Figs. 4 and 5.

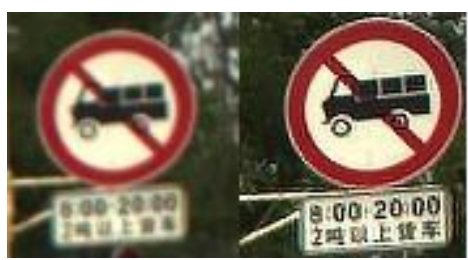

(a)

(b)

Fig. 4. Single traffic sign processing result (a) original image (b) image after enhance processing.

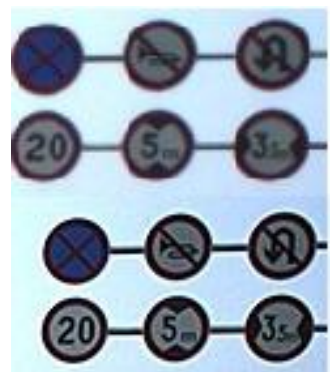

(a)

(b)

Fig. 5. Multiple traffic sign processing results. (a) original image (b) image after enhance processing.

\subsection{Improved Threshold Segmentation Algorithm}

Existing color image segmentation methods have two major problems: selecting the appropriate color space and selecting the appropriate segmentation. Segmentation algorithms can generate very few images for each type of segmentation. Therefore, to select the appropriate color space and segmentation algorithm, we must first analyze the image to be processed.

The method in this paper is applicable to moving images taken from a vehicle. Outdoor image processing is difficult a task because most of the external environment cannot be controlled, and weather and light have a huge impact on the extraction of traffic signs. The HSV color space is more similar to human eye color perception, and hue and saturation are not significantly affected by external light changes. So the proposed algorithm mainly applies segmentation in the HSV color space. 
Conversion from RGB color space to HSV color space is a simple and rapid nonlinear transformation. $H, S$, and $V$ are calculated using

$$
H=\left\{\begin{array}{cc}
\theta & , B \leq G \\
360-\theta & , B>G
\end{array}\right.
$$

where

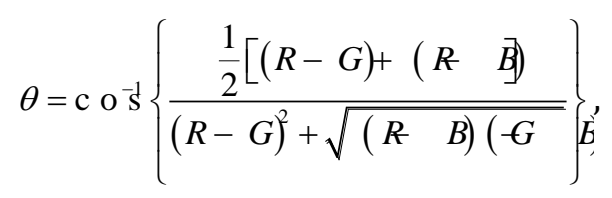

$$
\begin{aligned}
& S=1-\frac{[\min (R, G, B)]}{(R+G+B)},
\end{aligned}
$$

and

$$
V=\frac{1}{R+G+B} .
$$

We use these formulas to convert the image from RGB space to HSV space. The cameras are typically far away from road signs $(30-50 \mathrm{~m})$, so the traditional segmentation algorithm can break the target area. Severe road signs may not be detected. An increase in the saturation range can prevent breaks, but cannot remove issues caused by non-red backgrounds that lead to binary image complexities and increase the difficulty in the next step. To solve this problem, we propose an improved weighted RGB-HSV method. In this method,

$$
S^{\prime}=\frac{\min (R, G, B)}{\alpha \cdot R+\beta \cdot G+\gamma \cdot B}, \alpha+\beta+\gamma=1 .
$$

In the original formula

$$
\alpha=\beta=\gamma=\frac{1}{3} .
$$

That is, each color has the same weight, and we only filter or retain the image. In a large number of experimental results, it was easier to extract the target if we increased $\alpha$. We experimentally determined that $\alpha=0.5$ was best. The resulting optimal threshold value is shown in Table 1 .

Table 1. Optimal Threshold Value

\begin{tabular}{cccc}
\hline \hline & $H(0,360)$ & $S(0,1)$ & $V(0,1)$ \\
\hline Threshold value & $170<H<180$ & 0.5 & 0.5 \\
\hline \hline
\end{tabular}

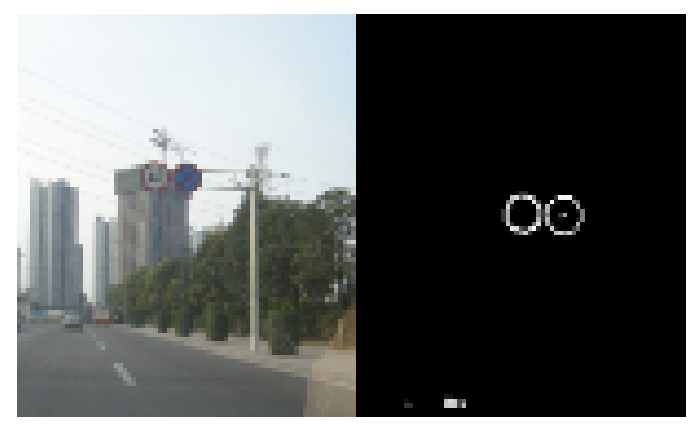

(a)

(b)

Fig. 6. Segmentation results (a).original image (b)segmentation result. 


\subsection{Morphological Filtering Process}

Images recorded in metropolitan areas can contain edge errors or highlights. To improve the accuracy, we used an opening operation to remove small details other than structural elements, keeping the overall gray value of the image and large bright regions sufficiently constant. This eliminated noise and interference. Closing operations also enhanced the image. That is, we used an open operation followed by a close operation to filter the entire image,

$$
g=I-\left(\begin{array}{ll}
b & )
\end{array}\right.
$$

and

$$
g=I \dashv(I \cdot)
$$

where $g$ is the output image; $B$ is the structuring element; and $I$ is the original image. The structural elements are shown in Fig. 8.

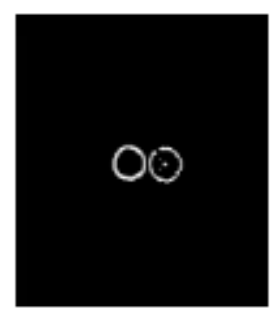

(a)

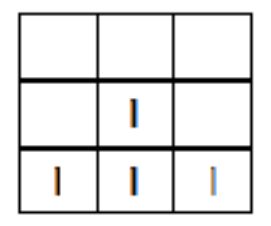

(b)

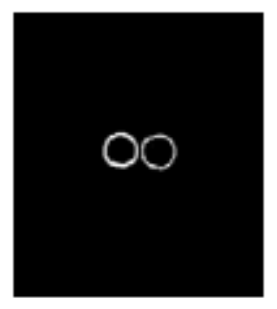

(c)

Fig. 8. Morphological processing: (a) original image; (b) structural element; and (c) result.

\section{Traffic sign detection}

Traffic sign detection methods are typically based on shape. In this paper, the detection method divides the binary image and applies a morphological processing step to determine the geometry of each connected region.

The algorithm is as follows.

Step 1: Extract the boundary of each region. If 4 neighboring pixels have a value of 1 , then the pixel point is set to 0 . This means that 1 is retained on boundary pixels.

Step 2: Obtain the individual properties of each connected region, that is, its center and external oval or circular axes.

Step 3: Use the center position to calculate signs that must be labeled. The axis length is used to calculate the circular characteristics of the connected region, that is, to determine its general shape. We then determine whether the sign is an image.

The circular features of a traffic sign are calculated using

$$
E=\frac{\max (W, H)}{\min (W, H)} .
$$

Here, $W$ and $H$ represent the signs horizontal and vertical axes. We investigated a large number of signs and determined the characteristic parameters shown in Table 2.

Table 2. Characteristic Parameter Table

\begin{tabular}{cll}
\hline \hline & Circular feature & Area feature \\
\hline Parameter value & $(1,1.3)$ & $(40,700)$ \\
\hline \hline
\end{tabular}

So we say that the image section is a sign if it conforms to the characteristics in Table 2. 


\section{Experimental Results and Analysis}

\subsection{Traffic Sign Detection Results}

We ran our experiments in the computer simulation environment MatlabR2010b, using a $3.4 \mathrm{GHz}$ processor and $8 \mathrm{~GB}$ of memory. The test sample was from in the national smart car challenge in 2015 to provide the open data set, which provided a dataset with various shooting parameters and conditions that degraded the image quality. The videos were recorded in natural environments. Each video consists of 10 to 18 image frames. The conditions included: sunny weather, normal illumination; sunny weather, strong light; and cloudy. Each frame contained $1280 \times 1024$ pixels. If detected in the final renderings can clearly indicate the sign area, counted correctly detected, if the image contains multiple signs, error detection, undetected, were not detected correctly. For each test situation, the correct detection rates were 95\%, 90\%, and 79\%, respectively. Compared with the traditional method, the correct detection rates has been greatly improved.

The results for this dataset are shown in Table 3.

Table 3. Detection Rate Statistics

\begin{tabular}{llcc}
\hline \hline Weather & $\begin{array}{l}\text { Number } \\
\text { of videos }\end{array}$ & $\begin{array}{l}\text { Traditional method } \\
\text { Detection rate }\end{array}$ & Detection rate \\
\hline Sunny, normal light & 100 & $90 \%$ & $95 \%$ \\
Sunny, strong light & 100 & $87 \%$ & $90 \%$ \\
Cloudy & 100 & $75 \%$ & $79 \%$ \\
\hline \hline
\end{tabular}

\subsection{Analysis of Results}

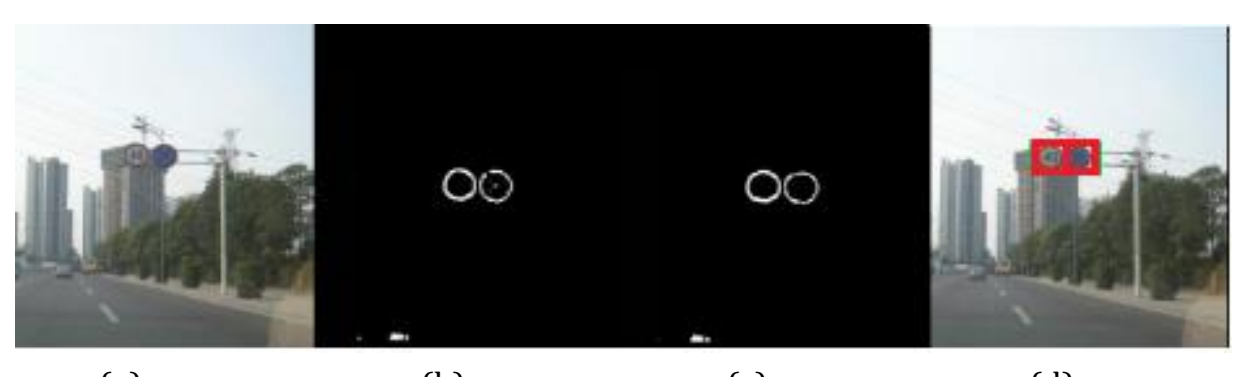

(a)

(b)

(c)

(d)

Fig. 8. Detecting multiple signs: (a) original image; (b) segmentation result; (c) image after morphological processing; and (d) detection result.

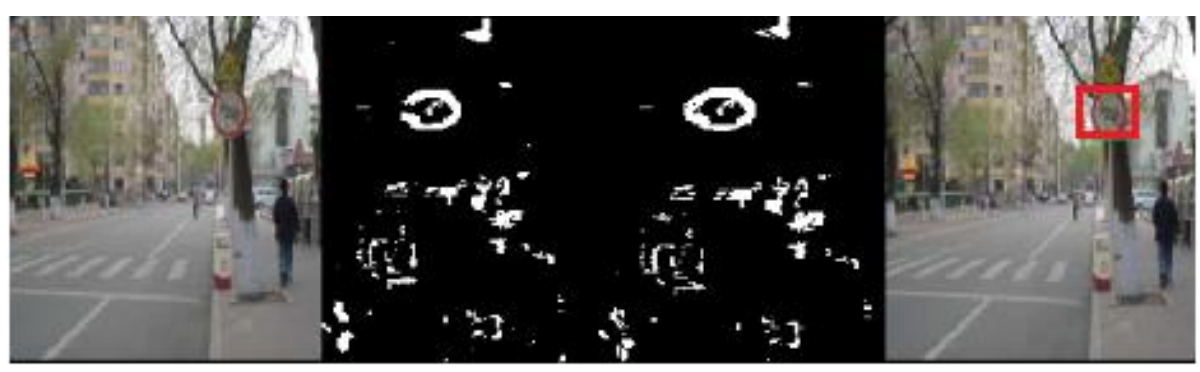

(a)

(b)

(c)

(d)

Fig. 9. Complicated background: (a) original image; (b) segmentation result; (c) image after morphological processing; and (d) detection result.

Fig. 8 is an image of a sign taken in normal weather conditions. From left to right are the original image, 
image segmentation, morphology processing result, and test result. These results show that the proposed algorithm accurately detected two signs. Fig. 9 is an image taken in cloudy conditions with a relatively complex background of willow branches. The image segmentation was not as effective as the previous example, but the morphological processing produced an accurate result. Thus, our method is very accurate when applied to images of various types of signs and conditions. It is also robust. Fig. 10 contains an image that has been degraded by motion blur. The proposed method produced good results.

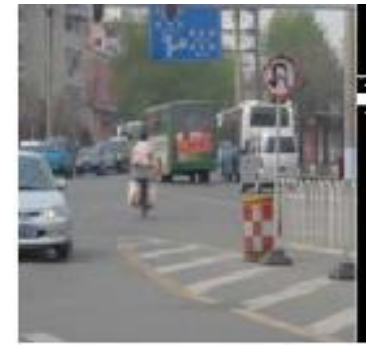

(a)

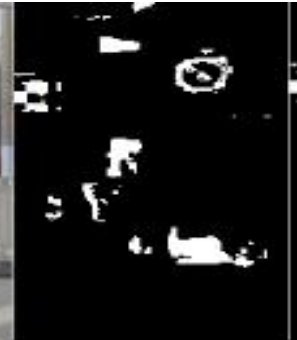

(b)

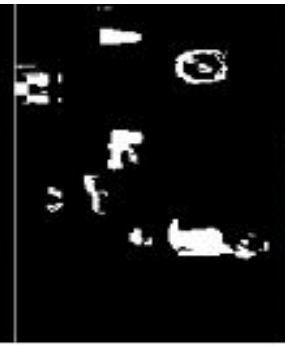

(c)

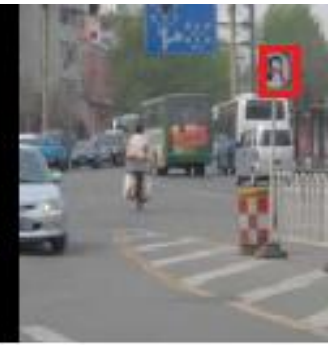

(d)

Fig. 10. Motion blur: (a) original image; (b) segmentation result; (c) image after morphological processing; (d) detection result.

Our method uses the improved Otsu segmentation algorithm [13] and an improved transformation algorithm to identify the sign area by considering the target area and shape characteristics. The discriminant function improved the accuracy compared with traditional methods based on colors. The technique does not traverse the entire image, which significantly reduced the operational time.

\section{Conclusion}

We proposed a color space conversion method that can more accurately identify red traffic signs. Furthermore, we can change the weighting coefficient to identify other colors. Our method improved the segmentation method compared with existing methods, and significantly reduced the computational time. By considering the regional and circular characteristics, it detects signs in images. Our experiments demonstrated that the method was highly accurate.

\section{Acknowledgment}

This work was supported by the National Natural Science Foundation of China (Grant Nos. 61370138, 61572077, 61271435, U1301251), Beijing Municipal Natural Science Foundation (Grant Nos. 4152017, 4162027).

\section{References}

[1] Wahyono, Kurnianggoro L., Hariyono, J., et al. (2015). Traffic sign recognition system for autonomous vehicle using cascade SVM classifier. Industrial Electronics Society, 4081-4086.

[2] Liu, C., Chang, F., Chen, Z., et al. (2016). Fast traffic sign recognition via high-contrast region extraction and extended sparse representation. IEEE Transactions on Intelligent Transportation Systems, 17(1), 79-92.

[3] Paclik, E., Novovicova, J., Somol, P., \& Pudil, P. (1999). Road Sign Classification using Laplace Kernel Classifier.

[4] Onat, E., \& Ozdil, O. (2015). Traffic sign classification using Hough transform and SVM. Proceedings of 2015 23th Signal Processing and Communications Applications Conference (SIU). IEEE.

[5] Mu-Dan, H. U., Yang, L. J., \& Zhu, S. D. (2009). Traffic sign segment based on chromatic aberration of 
three color-components. Mechanical \& Electrical Engineering Magazine.

[6] Escalera, A., Moreno, L. E., \& Salichs, M. A. (1997). Road traffic sign detection and classificatio. IEEE Transactions on Industrial Electronics, 44(6), 848859.

[7] Escalera, A., \& Aemingol, J. M. (2003). Traffic sign recognition and analysis for intelligent vehicles. Image and Vision Computing, 21(3), 247-258.

[8] Miguel, A. G., \& Manuel, O. D. (2012). Complete vision-based traffic sign recognition supported by an I2V communication system. Sensors, 12(2), 1148-1169.

[9] Loy, G., \& Barnes, N. (2004). Fast shape-based road sign detection for a driver assistance system. Proceedings of IEE RSJ International Conference on Intelligent Robots and Systems (vol. 1,pp. 70-75).

[10] Barnes, N., Zelinsky A. (2004). Real-time radial symmetry for speed sign detection. Proceedings of IEEE Intelligent Vehicles Symposium (pp. 566-571).

[11] Zhu, S. D., Liu, L. L., \& Lu, X. F. (2006). Color-geometric model for traffic sign recognition. Proceedings of IMACS Multiconference on Computational Engineering in Systems Applications (pp. 2028-2032).

[12] Paramanand, C., \& Rajagopalan, A. N. (2013).Motion blur for motion segmentation. IEEE International Conference on Image Processing (pp. 4244-4248) IEEE.

[13] Patel, J., Patwardhan, J., Sankhe, K., et al. (2011). Fuzzy inference based edge detection system using Sobel and Laplacian of Gaussian operators. Proceedings of Workshop on Emerging Trends in Technology (vol. 2, pp. 694-697). Mumbai, Maharashtra, India.

[14] Min, L., Luo, H. Y., Zheng, X. L., et al. (2012). Image segmentation based on improved Otsu algorithm. Journal of Nanjing University of Science \& Technology, 36(2), 332-337.

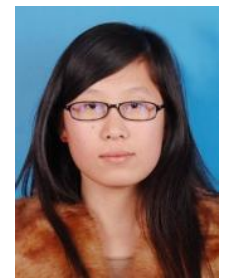

Qi Zhang was born in Xingtai, Hebei in 1996. She received her B.S. degree in communication engineering in 2014 at Tangshan College, Hebei, China. Currently, she is a postgraduate student from Beijing Key Laboratory of Information Services Engineering, Beijing Union University, China. Her research interests include digital image recognition and digital image segmentation.

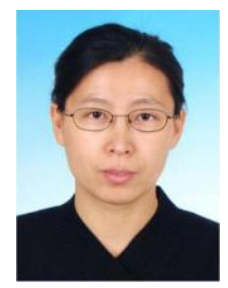

Ning He was born in Panjin, Liaoning Province in 1970. She graduated from the Department of Mathematics at Ningxia University in July 1993. She received M.S. degree and Ph.D. degree in applied mathematics from the Northwest University and Capital Normal University in July 2003 and July 2009, respectively. Currently she is a professor of the Beijing Union University. Her research interests include image processing and computer graphics.

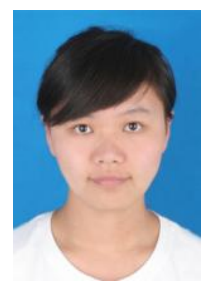

Yue Chi was born in Beijing, China in 1990.She received her B.S. Degree in electronic information engineering in 2013 at Beijing Union University, Beijing, China. Currently, she is a postgraduate student from Beijing Key Laboratory of Information Services Engineering, Beijing Union University, China. Her research interests include digital image in painting and digital image restoration. 